conditions. Use of human biological materials was approved by Institutional Review Board. Cortical and medulla tissues (average size; $0.5 \times 0.5 \times 0.2 \mathrm{~mm}$; width $\times$ length $\times$ thickness) from each patient were randomly allocated into 5 groups 1$)$ fresh control; 2) tissue decellularization using ionic detergent ( $0.5 \%$ sodium dodecyl sulfate [SDS]); 3) tissue decellularization using 1.0\% SDS; 4) tissue decellularization using non-ionic detergent $(1.0 \%$ Triton-X), and 5) tissue decellularization using 5.0\% Triton-X. After 24-hour decellularization process under various detergents, all tissues were treated with DNase 1 enzyme overnight and followed by detergent residual removal for another 24 hours. DNA concentration and detergent residuals from each tissue were evaluated using spectrophotometer (optical density 260/280 and $500 \mathrm{~nm}$, respectively).

Results: The major outcomes revealed that the lowest DNA concentration in cortical tissues was observed in $0.5 \%$ SDS group (group 2 vs. control, $229 \pm 101$ vs. $1,371 \pm 389 \mathrm{ng} / \mu \mathrm{L}$ ). In contrast to medulla, DNA content was effectively eliminated by $5.0 \%$ Triton-X (group 4 vs. control, $344 \pm 143$ vs. 1,820 \pm 431 ng/ $\mu \mathrm{L}$ ). However, detergent residuals were present in all treated tissues.

Conclusion: Ovarian scaffold might possibly be constructed either by ionic or non-ionic detergents which was depended on ovarian cell type. This technology could be further applied for in vitro follicle growth in young cancer patients who prefer to preserve their fertility.

Poster (M23)

Surgical Techniques \& Perioperative Management

https://doi.org/10.3802/jgo.2021.32.S1.M23

\section{Fluorescence ureter navigation during laparoscopic hysterectomy}

\section{Pinyada Panyavaranant, "Trainee Manchana}

Gynecologic Oncology Unit, Obstetrics \& Gynecology Department, Faculty of Medicine, King Chulalongkorn Memorial Hospital, Bangkok, Thailand (pinnpanya@gmail.com)

Recently, laparoscopic surgery has been widely employed for gynecological surgery including total laparoscopic hysterectomy. However, laparoscopic surgery may injure the ureters slightly increase urological tract injury thus leading to long-term medical complications such as fistula formation and renal failure, resulting in the need for additional treatments and increase morbidity. Nonetheless, such complications can be prevented by knowing direct visualization the location of ureters throughout the whole surgery session and carefully not to injure the ureters whether during the cutting, coagulation, or ligation. Identification of the ureters can be done by insertion of fluorescence ureteric catheters through cystoscopy prior to the surgery. Afterwards, using a laparoscope that is capable of absorbing the near-infrared spectral range together with the fluorescence imaging system, the inserted ureteric catheters are then illuminated. This procedure enables the surgeons to see the ureters clearly and thus facilitates safe surgery.

Poster (M24)

Surgical Techniques \& Perioperative Management

https://doi.org/10.3802/jgo.2021.32.S1.M24

\section{Inguinofemoral lymph node dissection for vulvar cancer}

\section{Mikio Mikami}

Department of Obstetrics and Gynecology, Tokai University School of Medicine, Isehara, Japan (mmikami@is.icc.u-tokai.ac.jp)

The skills and techniques of the surgery for vulvar cancer are derived from the traditional surgery. Especially, the inguinal lymph node dissection is an essential part of the surgery but is not common as a daily practice. It is important for a surgeon to be well acquainted before surgery with the anatomy of the femoral triangle and the skills of the dissection before surgery. I will show you the standard procedure of Inguinofemoral lymph node dissection. Before dissecting the femoral area, surgeon must identify the position of femoral artery by touching and feeling the pulse and imaging the anatomical structures beneath the fascia lata.

Poster (M25)

Gynecologic Pathology, Genetics and Epidemiology https://doi.org/10.3802/jgo.2021.32.S1.M25

\section{Genomic landscape and its correlations with immunotherapy-related biomarkers in Chinese gynecologic cancer patients}

\author{
Guoliang Li, Chaoran Xia, Shoutai Ding, Pushan Shi, \\ Zhixiang Yan, Peng Luo, Jiajia Xu* \\ Topgen Biological Medicine Technology Co., Ltd., Shanghai, China \\ (xujiajia@topgen.com.cn)
}

Objective: To explore mutational characterization, tumor mutational burden (TMB) and its correlations with gene alterations, TMB in Chinese gynecologic cancer.

Methods: In 207 samples (formalin-fixed, paraffin-embedded tissue samples) including 141 ovarian cancer (OC), 28 cervical cancer (CC) and 38 endometrial carcinoma (EC), were detected by OncoDrug-Seq 603-gene panel assay through next generation sequencing (NGS) using Illumina NovaSeq 6000.

Results: In 207 samples, the median TMB was 4.7/Mb with the range from $0 / \mathrm{Mb}$ to $248 / \mathrm{Mb}$. The median TMB of OC, CC, 
and $\mathrm{EC}$ was $4.7 / \mathrm{Mb}, 4 / \mathrm{Mb}$ and $4.7 / \mathrm{Mb}$, respectively. The top 10 genes with mutational incidence were TP53 (42.5\%), PIK3CA (16.4\%), ARID1A (11.6\%), PTEN (11.1\%), KRAS (9.7\%), BRCA1 (7.7\%), BRCA2 (5.8\%), KMT2D (4.3\%), RAD50 (3.9\%), KMT2C (3.9\%). Specially, mutational incidence differed in mutational incidence and spectra for genes. With RAD51D (3.5\%) in OC group; TERT (10.7\%), EGFR (7.1\%), FANCA (3.6\%) and PDE4DIP (7.1\%) in CC group; EGFR (3.5\%), FANCA (13.2\%), and CTCF (10.5\%) in EC group, respectively. While TP53, PIK3CA, PTEN, $K R A S, B R C A 2$ appeared to co-occurrent more likely in OC, CC, and EC samples, BRCA1 and KMT2C showed higher frequency in OC group, RAD50 appeared more likely in EC group, ARID1A appeared more likely in OC and EC groups, KMT2D appeared in more likely in OC and CC groups. Furthermore, our data showed that median TMB had no significant differences among OC, CC, and EC. But the arrays of the top 10 were displayed similarly and specifically, which can be potential biomarkers for target-therapy.

Conclusion: Differences observed in the mutation spectra for spontaneous gynecologic tumors and similarities in spectra support the concept that mutation spectra can serve as a "fingerprint" of each type of cancer. 